\title{
Percepción de los riesgos de su puesto de trabajo de los médicos internos residentes de un hospital secundario
}

\author{
M. Reyes Martín-Prieto, M. Fe Gamo, M. Josefa Ruiz-Figueroa
}

Introducción. La formación sanitaria especializada ('residencia') es un período crucial para el desarrollo profesional posterior. Pretendimos objetivar cuáles son los riesgos que más preocupan a los residentes de nuestro hospital y compararlos con los resultados de la evaluación de riesgos realizada en sus puestos de trabajo para poder plantear estrategias que nos ayuden en nuestra práctica diaria.

Sujetos y métodos. Estudio descriptivo en la población de 83 residentes que se incorporaron al hospital durante los años 2005-2007. Se obtuvieron los datos de una encuesta, revisando sus historias clínico-laborales y la evaluación de riesgos. Se realizó un análisis descriptivo de estas variables, se compararon los resultados de la encuesta con la evaluación por los técnicos y se analizó la posible asociación de la percepción del riesgo con la especialidad o el año de residencia mediante el programa estadístico Stata.

Resultados. Los riesgos más referidos son las radiaciones ionizantes y los psicosociales, seguidos de los biológicos y ergonómicos, aunque varía a lo largo de la residencia. Existen discrepancias muy significativas entre lo que refieren los encuestados y la evaluación de riesgos de los técnicos.

Conclusiones. La percepción de los riesgos laborales en los residentes no se corresponde con las condiciones de trabajo objetivadas por los técnicos en la mayoría de los riesgos valorados por diferentes razones. Desarrollar un programa formativo específico para este colectivo les ayudaría a tener un conocimiento más realista. Algunos riesgos requerirían una reevaluación por la parte técnica tomando en consideración la percepción de la situación de riesgo.

Palabras clave. Factores de riesgo. Formación médica especializada. Percepción del riesgo. Residencia.

\section{Occupational risks perception in medical internal residents of a secondary hospital}

Introduction. The medical specialized training 'residence' is a critical period for the later professional development. We tried to determine which risks the residents perceive in their activities and to compare them with the risk evaluation developed in their jobsites, to design oriented strategies that help us in daily practice.

Subjects and methods. We have implemented a descriptive study whose targeted population was 83 residents who entered in the hospital along the period 2005-2007. Data was obtained through a survey specifically designed and their clinical occupational files -including the risk evaluation-. We have made a descriptive analysis of personal perception and technical evaluation data and verified its relationships with some theoretical determinant factors like year of residence or medical specialty.

Results. The risks mainly perceived by residents are: ionizing radiation and psychosocial ones and, secondly, biological and ergonomic risks. We evidence a gap between the survey's results and the risk evaluation data linked to different factors. Occupational risks perception changes along the years of training residence keeping different trend models (increasing or decreasing the gap) according to different types of risk.

Conclusions. In most of situations residents' occupational risks perception does not correspond directly with their objective work conditions. Developing a formative program specifically addressed to these workers would help them to better recognise and manage. Some risks should be reassessed by technicians taking into account the analyzed group's perception.

Key words. Education medical graduate. Internship and residency. Risk factors. Risk perception.

Servicio de Prevención; Área de Medicina del Trabajo; Michelín España-Portugal; Aranda de Duero, Burgos (M.R. Martín-Prieto). Escuela Nacional de Medicina del Trabajo; Instituto de Salud Carlos III; Madrid (M.F. Gamo). Servicio de Prevención de Servicios Centrales; Ministerio de Sanidad y Política Social; Madrid, España (M.J. RuizFigueroa).

Correspondencia:

Dra. M. Reyes Martín Prieto. Servicio de Prevención, Área de Medicina del Trabajo. Michelín España-Portugal. Avda. Luis Mateos, s/n. Apdo. 151. E-09400 Aranda de Duero (Burgos).

E-mail:

rmartinprieto@yahoo.es

Agradecimientos:

A la Escuela Nacional de Medicina del Trabajo de Madrid y la Unidad Docente del Principado de Asturias.

Este trabajo formó parte del Programa Científico de Formación MIR de Medicina del Trabajo desarrollado por la Escuela Naciona de Medicina del Trabajo del Instituto de Salud Carlos III, en convenio con la Unidad Docente de Medicina del Trabajo del Principado de Asturias.

(c) 2011 Educación Médica 


\section{Introducción}

En el hospital trabajan distintos profesionales con diversos niveles de conocimientos, habilidades y tareas. La formación médica especializada en España se basa en un sistema denominado de 'residencia', período en el cual el médico interno residente (MIR), futuro especialista, adquirirá conocimientos, habilidades y actitudes, además de la responsabilidad profesional, que le permitirá el desarrollo autónomo del trabajo. Desde el punto de vista de la prevención son un colectivo heterogéneo sometido a riesgos laborales de muy diversa índole. Esquemáticamente podemos clasificar los riesgos en función de la especialidad técnica preventiva que lo estudia $[1,2]$ :

- Riesgos relacionados con la higiene:

a) Riesgos químicos: contaminantes en forma de polvos, gases, irritantes, mutágenos, cancerígenos y sensibilizantes que pueden actuar por contacto o inhalación.

b) Riesgos físicos: exposición a ruido/vibraciones, radiaciones ionizantes o no ionizantes.

c) Riesgos biológicos: exposición a virus, hongos y bacterias ya sea por contacto (salpicadura...), inhalación o punción percutánea a fluidos biológicos.

- Riesgos para la seguridad: riesgos de incendio, eléctricos o mecánicos (golpes y caídas).

- Riesgos de ergonomía: disconfort ambiental por inapropiada temperatura o iluminación, carga física de trabajo, posturas forzadas (utilización de pantallas de datos...).

- Riesgos psicosociales: carga de trabajo mental y psíquica (en relación con trabajo a turnos, trabajo nocturno, atención al paciente y sus familiares, riesgo de agresiones, etc.).

Estos riesgos que se detectan y registran en la evaluación de riesgos de los puestos de trabajo constituyen el punto de partida para las actividades preventivas que tratarán de conseguir la seguridad y salud de estos trabajadores, incluyendo las actividades de formación en materia de riesgos laborales. Esta formación puede y debería incluirse en el proceso formativo del MIR, pero, de hecho, no se contempla de forma sistemática y normalizada. Por otra parte, se suele poner el énfasis en la promoción de la cultura preventiva y en el desarrollo de habilidades de 'trabajo seguro' para lograr una prevención eficaz. Y éstas radican, a su vez, en acciones formativas cuya incidencia variará según actitudes y comportamientos que se derivan inexcusablemente de la conciencia de estar en peligro, es decir, de la percepción subjetiva del riesgo. Sin ese primer factor, imprescindible, es de esperar que las acciones dirigidas a la formación en prevención no calen ni lleguen a traducirse en pautas de trabajo seguro.

Los MIR constituyen un colectivo numeroso del que no hemos encontrado estudios (se realizó una estrategia de búsqueda en las bases de datos de PubMed y CisDoc, utilizando las palabras clave resident doctors, medical postgraduate y training physician, pero no se encontraron artículos vigentes o útiles) que trataran la percepción individual del riesgo y sus posibles modificaciones en el tiempo, especialmente en un período que parece crucial en la promoción de la cultura de la prevención: el ingreso como trabajador, frecuentemente joven e inexperto, y su evolución en pleno proceso de formación y adaptación al ejercicio de la medicina [3-6].

Hemos pretendido en este estudio objetivar cuáles son los riesgos que más preocupan a los residentes de nuestro ámbito y compararlos con los riesgos detectados en la evaluación de riesgos realizada por los técnicos en sus puestos de trabajo, con el fin de plantear oportunidades de mejora en nuestra actividad diaria del Servicio de Prevención de un hospital secundario.

\section{Sujetos y métodos}

La población de estudio la constituyen los 83 MIR que ingresaron en los años 2005, 2006 y 2007 para formarse en un hospital secundario, y a los que el Servicio de Prevención realizó el reconocimiento médico-laboral inicial. A todos ellos se les envió en el año 2008 el cuestionario elaborado a propósito (Anexo), que contestaron 45 residentes, lo que representa el 54\% de la población de estudio. Esto supondría, si se tratara de una muestra aleatoria, un error muestral de $\pm 10 \%$ para $p>0,05$. Los datos se obtuvieron, por tanto, de dos fuentes:

- Una encuesta anónima, enviada a cada uno de ellos por correo interno y por correo electrónico, que recogía variables sociodemográficas, laborales y un cuestionario sobre su percepción de los riesgos en su actividad laboral.

- Las historias clínico-laborales de todos estos residentes, incluyendo las evaluaciones de riesgos de los puestos de trabajo hechas por los técnicos del servicio; para este último grupo de variables sólo se procesaron las relativas a los puestos de trabajo de los residentes que contestaron a la encuesta.

Se grabaron los datos en hojas de cálculo Excel cuyo análisis y las correspondientes tablas de frecuencias relativas y absolutas se obtuvieron mediante el pro- 
grama estadístico EPI Info. Se realizó un análisis descriptivo de las variables y, a continuación, un análisis bivariante con el programa estadístico Stata: hemos efectuado una comparación de proporciones entre la percepción del riesgo por los residentes y la detección por los técnicos en la evaluación y hemos analizado la asociación entre las variables relativas a la percepción y el año de residencia o el tipo de especialidad médica como factores explicativos. La estimación de proporciones de las distintas variables estudiadas se realizó con intervalos de confianza del 95\%, y la comparación de proporciones, con chi al cuadrado.

Consideramos como limitaciones del estudio posibles sesgos de autoselección y de información a la hora de realizar la encuesta. Para su control hemos procurado que las preguntas de la encuesta fueran lo más concretas posibles y así evitar los sesgos de memoria, y hemos comprobado que los factores personales (sexo y edad) y los relativos a los puestos de trabajo (especialidad y año de residencia) no difieren entre la población $(n=83)$ y la muestra encuestada $(n=45)$ (Tabla I). Un factor a valorar como causa de la baja tasa de respuesta pudo ser la dificultad de localizar a varios de los residentes que se encontraban rotando tanto en unidades docentes diferentes a la de referencia como en dispositivos externos de ésta.

En definitiva, hemos trabajado con una muestra autoseleccionada, interesada en participar y cuyo ámbito de referencia han sido, preferentemente, las unidades docentes localizadas en el mismo hospital. Por tanto, los resultados, a priori, podrían haber sobreestimado la percepción de riesgo - por exceso (relativo) de interés- y pueden aparecer sobrerrepresentados los MIR del entorno próximo de trabajo, de mayor interés para la práctica del Servicio de Prevención.

\section{Resultados}

La tabla II presenta de forma sintética la valoración que los residentes hacen de diferentes aspectos de sus condiciones de trabajo, como adecuadas o incorrectas, y cuáles de éstos los perciben como 'riesgo.' De hecho, trata de confrontar la experiencia de 'disconfort' frente a la conciencia de riesgo. Así, mientras las condiciones de trabajo relacionadas con la ergonomía se señalan con más frecuencia -en la mitad de las respuestas- como molestas o 'incorrectas', no son esos aspectos los que más se señalan como riesgo, sino las radiaciones ionizantes, la carga mental y la psíquica para seis de cada siete residentes.
Tabla I. Diferencias entre los residentes que contestaron o no a la encuesta.

\begin{tabular}{|c|c|c|c|c|c|c|}
\hline & \multicolumn{2}{|c|}{$\begin{array}{l}\text { Residentes que } \\
\text { contestaron }\end{array}$} & \multicolumn{2}{|c|}{$\begin{array}{l}\text { Residentes que } \\
\text { no contestaron }\end{array}$} & \multirow{2}{*}{$\begin{array}{c}\text { Población } \\
\text { diana total }\end{array}$} & \multirow[t]{2}{*}{$p$} \\
\hline & $n$ & $\%$ & $n$ & $\%$ & & \\
\hline \multicolumn{7}{|l|}{ Sexo } \\
\hline Varones & 7 & 15,00 & 8 & 21,00 & 15 & 0,76 \\
\hline Mujeres & 38 & 85,00 & 30 & 78,90 & 68 & 0,51 \\
\hline \multicolumn{7}{|l|}{ Edad } \\
\hline$\leq 32$ años & 33 & 73,33 & 27 & 71,05 & 60 & 0,85 \\
\hline 33-39 años & 9 & 20,00 & 7 & 18,42 & 16 & 0,94 \\
\hline$\geq 40$ años & 3 & 6,67 & 4 & 10,52 & 7 & 0,86 \\
\hline \multicolumn{7}{|l|}{ Año de residencia } \\
\hline Primero & 13 & 28,90 & 16 & 42,10 & 29 & 0,46 \\
\hline Segundo & 21 & 46,70 & 6 & 15,78 & 27 & 0,17 \\
\hline Tercero & 11 & 24,40 & 16 & 42,10 & 27 & 0,34 \\
\hline
\end{tabular}

\section{Especialidad}

\begin{tabular}{lcccccc} 
Atención primaria & 21 & 46,70 & 15 & 39,47 & 36 & 0,43 \\
$\begin{array}{l}\text { Especializada médica } \\
\begin{array}{l}\text { Especializada } \\
\text { médico-quirúrgica }\end{array}\end{array}$ & 12 & 26,70 & 5 & 13,15 & 17 & 0,54 \\
Otras & 7 & 11,10 & 12 & 31,57 & 17 & 0,38 \\
\hline
\end{tabular}

Total

45

38

83

\section{Percepción de riesgos (encuesta) \\ frente a riesgos detectados \\ en la evaluación de riesgos}

Al comparar la frecuencia con que se valora subjetivamente esos aspectos y la proporción de puestos en los que los técnicos los han identificado como 'riesgo' (Tabla III) se aprecia una falta de correspondencia que es estadísticamente significativa en ocho de los diez riesgos considerados y especialmente en 'radiaciones ionizantes', 'contacto químico' y 'carga psíquica', que los residentes los refieren como riesgo muy por encima de lo reflejado en las evaluaciones. En sentido contrario, los riesgos biológicos que los evaluadores identifican en todos los puestos de trabajo sólo son referidos por dos de cada tres o tres de cada cuatro residentes. 
Tabla II. Percepción global de las condiciones de trabajo y los factores de riesgo en su puesto de trabajo de los residentes encuestados.

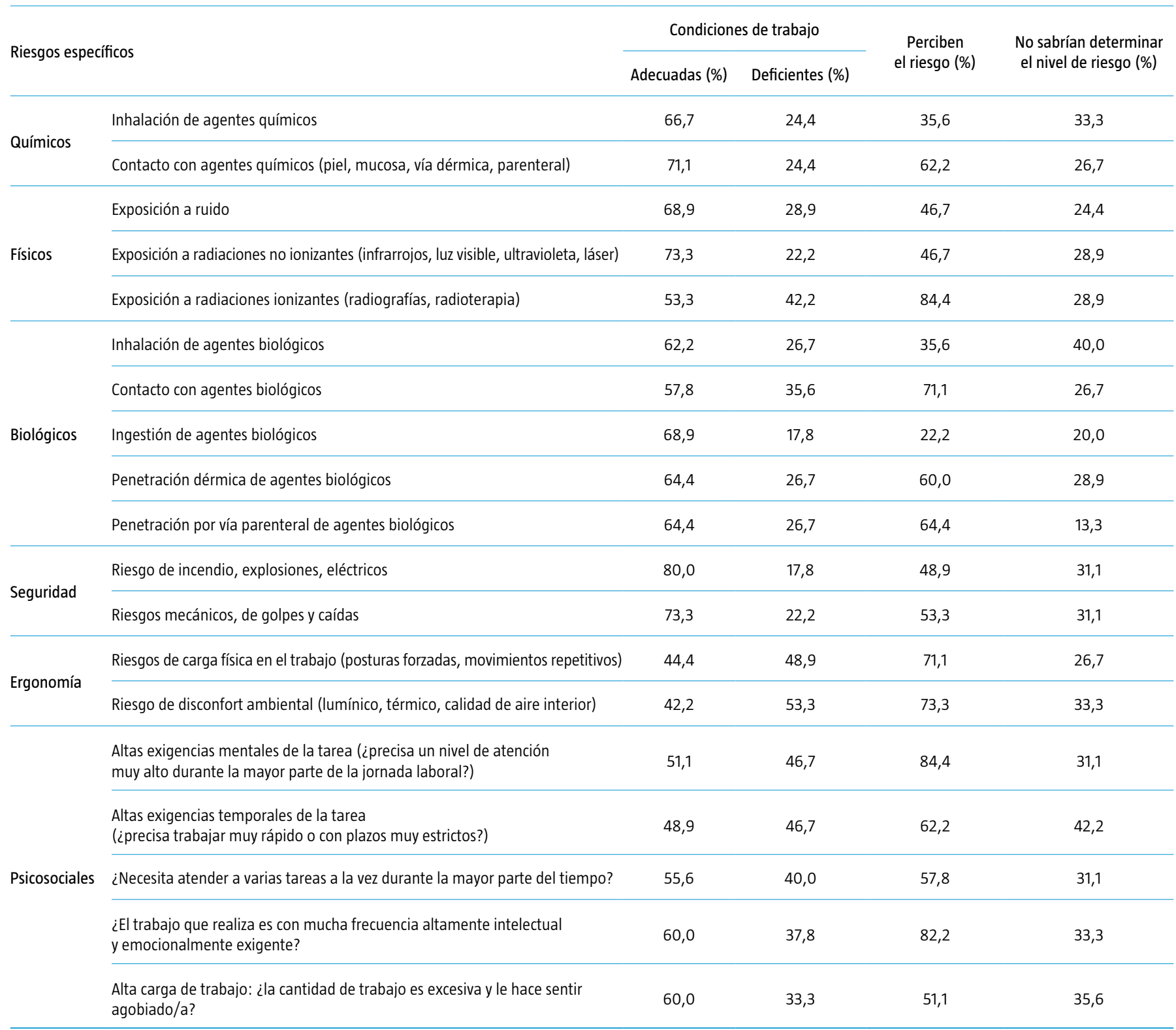

Percepción (encuesta) frente

a evaluación de riesgos en

función del año de residencia

La figura representa la evolución de la percepción del riesgo a lo largo de la residencia, comparando los diferentes resultados con la evaluación hecha por los técnicos como referente. Se trataba de reflejar cómo la experiencia laboral va cambiando la per- cepción de riesgo respecto a algunas condiciones de trabajo. Cabe destacar que algunos riesgos se perciben con mayor frecuencia a medida que avanza la residencia: la 'carga mental' y el 'riesgo biológico parenteral' que se acercan, en ese proceso, a lo evaluado por los técnicos, y el 'ruido' y la 'carga psíquica', que también con una percepción creciente, sin embargo, se alejan de lo evaluado. En sentido contrario, dos riesgos aparecen menos percibidos al final que 
Figura. Percepción (encuesta) frente a evaluación de riesgos en función del año de residencia.

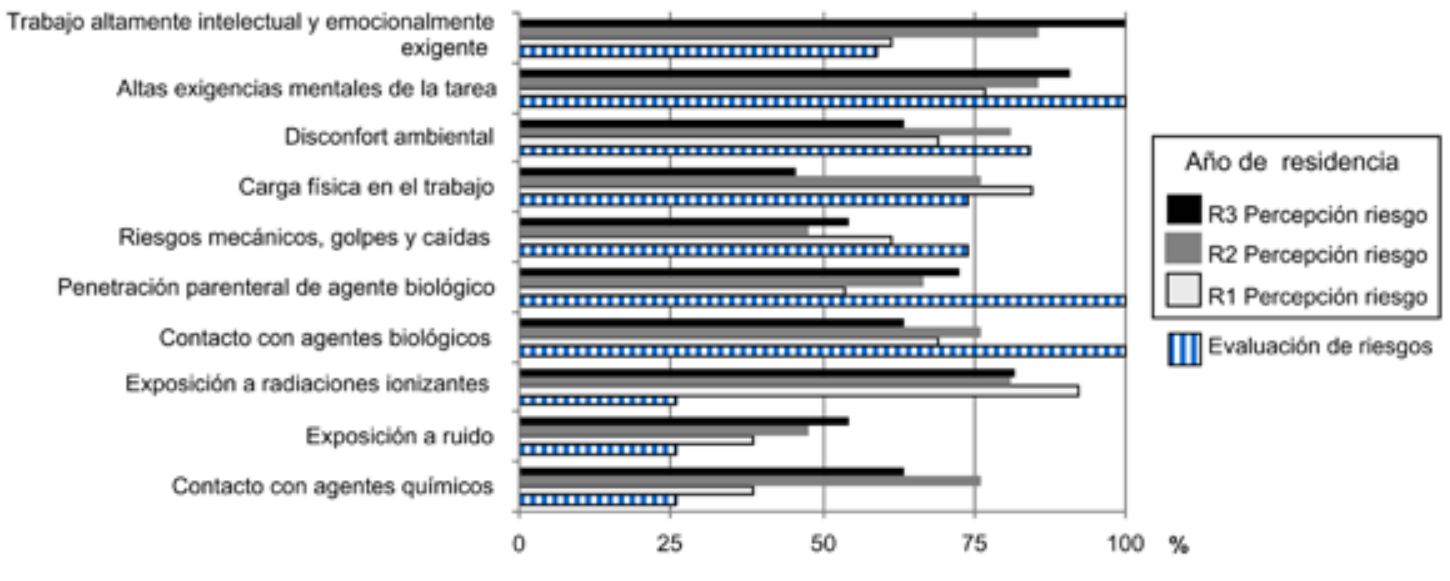

al inicio de la residencia: las 'radiaciones ionizantes' y la 'carga física', aunque el primero se sigue percibiendo con mayor frecuencia de la que se identifica en la evaluación mientras que en el segundo caso se acerca progresivamente a lo evaluado.

\section{Percepción (encuesta) frente}

a evaluación de riesgos en

función de la especialidad elegida

Dado que el tipo de especialidad en la que se forma el MIR determina sus condiciones de trabajo y los riesgos específicos de sus puestos, hemos estratificado la muestra por los grupos de especialidad que, de hecho, corresponden a perfiles homogéneos de evaluación de riesgos. En la tabla IV se evidencia en qué riesgos residen las discrepancias fundamentales entre percepción y evaluación. Resultan paradójicos algunos datos: en todos los riesgos higiénicos (detectables mediante métodos e instrumentos objetivos), la discordancia es tan importante como que todos los MIR de especialidades de primaria y tres de cada cuatro de los de especialidades médicas perciben el riesgo de radiaciones ionizantes, mientras que los de especialidades en los que los técnicos sí identifican la posibilidad de exposición, sólo la mitad lo perciben como riesgo laboral. En el otro extremo, la percepción de sufrir por 'carga psíquica' (riesgo que se ha de evaluar con la participación del trabajador), la discrepancia evidencia que entre los puestos en que la evaluación no lo señala, cuatro de cada cinco MIR de especialidades médicas lo perciben como riesgo.

\section{Discusión}

Tras una búsqueda dirigida en las principales bases de documentación científica para poder comparar los resultados obtenidos, sólo hemos encontrado un estudio que tratara de forma específica la percepción de los riesgos propios de su puesto de trabajo entre los MIR, en Australia, con estudiantes junto a residentes, y dedicado a un único factor de riesgo: significativamente, las radiaciones ionizantes [7]. Conscientes de esta limitación, hemos objetivado nuestra situación para plantear oportunidades de mejora en nuestra actividad diaria del Servicio de Prevención de un hospital secundario. La tasa de respuesta obtenida $(54 \% ; n=45)$ es menor que en otros estudios $[8,9]$ y nuestra muestra presenta características personales y profesionales similares a las de otros estudios de MIR [10].

Los riesgos percibidos con más frecuencia coinciden con los riesgos prevalentes según los manuales consultados acerca de riesgos laborales en trabajadores sanitarios [1,2]. Sin embargo, existen diferencias llamativas entre lo que contestan los residentes en la encuesta de percepción y lo estimado en la evaluación de riesgos en lo referente a riesgo de contacto con agentes químicos y exposición a radiaciones ionizantes, que son entre dos y tres veces más frecuentemente percibidos que evaluados. La alta percepción frente a los riesgos químicos coincide con diversas fuentes que hablan de la importancia de estudiar estos agentes en el ámbito laboral [11]. En relación a las radiaciones ionizantes, la percepción disminuye según progresa la residen- 
Tabla III. Comparación entre riesgos percibidos (encuesta) frente a los riesgos identificados por los técnicos en la evaluación de riesgos.

\begin{tabular}{lcc}
\hline & $\begin{array}{c}\text { Percepción de riesgos } \\
\text { según encuesta }\end{array}$ & $\begin{array}{c}\text { Riesgos identificados en } \\
\text { la evaluación de riesgos }\end{array}$ \\
\hline Contacto con agentes químicos & $62,2(47-76)$ & $26,7(13-38)$ \\
\hline Exposición a ruido & $46,7(31-60)$ & $26,7(13-38)$ \\
\hline Exposición a radiaciones ionizantes & $84,4(73-94)$ & $26,7(13-38)$ \\
\hline Contacto con agentes biológicos & $71,1(57-84)$ & $100(100)$ \\
\hline Penetración parenteral de agentes biológicos & $64,4(49-78)$ & $100(100)$ \\
\hline Riesgos mecánicos, golpes y caídas & $53,3(38-67)$ & $73,3(60-85)$ \\
\hline Carga física en el trabajo & $71,1(57-84)$ & $73,3(60-85)$ \\
\hline Disconfort ambiental & $73,3(60-85)$ & $84,4(73-94)$ \\
\hline Altas exigencias mentales de la tarea & $84,4(73-94)$ & $100(100)$ \\
\hline $\begin{array}{l}\text { Trabajo altamente intelectual } \\
\text { y emocionalmente exigente }\end{array}$ & $82,2(70-93)$ & $46,7(31-61)$ \\
\hline
\end{tabular}

Entre paréntesis, intervalo de confianza.
Los riesgos biológicos, tanto el contacto como la posibilidad de penetración parenteral, son infravalorados por quienes contestan la encuesta en comparación con la estimación de los técnicos que identifican el riesgo en todos los puestos. Creemos que podría deberse a una sensación de 'falsa seguridad' en aquello que parece más conocido [14], lo que se apoyaría en el dato de que la percepción del riesgo biológico parenteral aumenta a medida que progresa la residencia.

Consideramos que la alta frecuencia de percepción de riesgos ergonómicos está en consonancia con el uso generalizado de las nuevas tecnologías (pantallas de visualización de datos, etc.) en la realización de su trabajo diario, cuyos riesgos son más conocidos. Es de resaltar que la percepción de la carga física disminuye de año en año.

Creemos que organizar cursos de formación específicos para este colectivo y realizar un protocolo de acogida-guía a partir de los datos que hemos obtenido en este estudio ayudaría a un mayor conocimiento real de los riesgos laborales y quizás a una mayor sensibilidad a la prevención. Por otro lado, otros riesgos requieren una reevaluación por nuestra parte para adaptarnos a los riesgos emergentes (ergonómicos y psicosociales). La elaboración de listados para cumplimentar activamente por los residentes en los reconocimientos, incluyendo los riesgos que consideremos más relevantes, con la descripción de las tareas que realizan (manipulación de sustancias químicas o biológicas, etc.), contribuiría en parte a subsanarlo y proporcionaría datos de alta sensibilidad para la reevaluación de sus puestos de trabajo.

En conclusión, la formación médica especializada es un período crucial en el que se adquieren hábitos que permanecerán en la vida laboral del futuro especialista y, en ese sentido, para poder desarrollar una prevención de riesgos laborales más eficaz y que llegue a todos los médicos se debería tener en cuenta que:

- La percepción de los riesgos a los que están expuestos los residentes en su puesto de trabajo no se corresponde directamente con la situación objetiva de trabajo.

- Al avanzar la residencia, el conocimiento de la situación de trabajo y la percepción de los riesgos varía respecto a los momentos iniciales como parte del proceso formativo.

- Riesgos en los que es imprescindible la opinión del trabajador para su evaluación muestran una discrepancia significativa entre percepción y evaluación, lo que sugiere la necesidad de reconsiderar los métodos de evaluación específicos. 
Tabla IV. Percepción de los riesgos (encuesta) frente a evaluación de riesgos según la especialidad.

\begin{tabular}{|c|c|c|c|c|c|c|}
\hline & \multicolumn{6}{|c|}{ Tipo de especialidad } \\
\hline & \multicolumn{2}{|c|}{ Primaria $(n=21)$} & \multicolumn{2}{|c|}{ Médica $(n=12)$} & \multicolumn{2}{|c|}{$\begin{array}{l}\text { Médico-quirúrgica } \\
\text { y otras }(n=12)\end{array}$} \\
\hline & $\begin{array}{l}\text { Percepción } \\
\text { (encuesta) }\end{array}$ & $\begin{array}{c}\text { Riesgo } \\
\text { identificado }\end{array}$ & $\begin{array}{l}\text { Percepción } \\
\text { (encuesta) }\end{array}$ & $\begin{array}{c}\text { Riesgo } \\
\text { identificado }\end{array}$ & $\begin{array}{l}\text { Percepción } \\
\text { (encuesta) }\end{array}$ & $\begin{array}{c}\text { Riesgo } \\
\text { identificado }\end{array}$ \\
\hline Contacto con agentes químicos & 66,67 & No & 66,67 & No & 50,00 & Sí \\
\hline Exposición a ruido & 52,38 & No & 33,33 & Sí & 50,00 & No \\
\hline Exposición a radiaciones ionizantes & 100,00 & No & 75,00 & No & 66,70 & Sí \\
\hline Contacto con agentes biológicos & 57,14 & Sí & 83,33 & Sí & 83,30 & Sí \\
\hline $\begin{array}{l}\text { Penetración parenteral } \\
\text { de agentes biológicos }\end{array}$ & 57,14 & Sí & 75,00 & Sí & 66,70 & Sí \\
\hline Riesgos mecánicos, golpes y caídas & 61,90 & Sí & 58,33 & No & 33,30 & Sí \\
\hline Carga física en el trabajo & 80,95 & Sí & 50,00 & No & 66,70 & Sí \\
\hline Disconfort ambiental & 71,43 & Sí & 66,67 & Sí & 83,30 & Sí \\
\hline Altas exigencias mentales de la tarea & 80,95 & Sí & 83,33 & Sí & 91,70 & Sí \\
\hline $\begin{array}{l}\text { Trabajo altamente intelectual } \\
\text { y emocionalmente exigente }\end{array}$ & 95,24 & Sí & 83,33 & No & 50,00 & No \\
\hline
\end{tabular}

- Consideramos prioritario el desarrollo de un plan específico de formación en prevención para este colectivo y la reevaluación de aquellos riesgos en los que la discrepancia se ha evidenciado como especialmente relevante y consistente a lo largo del tiempo de residencia.

\section{Bibliografía}

1. Yassi A, Warshaw L. Naturaleza del sector de la asistencia sanitaria. Cap. 97: Asistencia sanitaria: naturaleza y problema de la salud en el trabajo. Enciclopedia de la Organización Internacional del Trabajo [monografía en Internet], 3 ed. Madrid: Ministerio de Trabajo e Inmigración; 1998. URL: http://www.insht.es/InshtWeb/Contenidos/Documentacion/ TextosOnline/EnciclopediaOIT/tomo3/97.pdf. [31.07.2009].

2. Zafra JA, Novalbos JP, Costa MJ, Moreno JJ. Evaluación de riesgos en el hospital. In Gestal-Otero J.J. Riesgo del trabajo del personal sanitario. 3 ed. Madrid: InteramericanaMcGraw Hill; 2003. p. 141-58.

3. Garnés-Ros A. Estudio de las fuentes de estrés laboral en médicos residentes. Revista Centro de Salud Hospital General Universitario de Alicante 2001: 568-72.

4. Escribá-Agüir V, Bernabé-Muñoz Y. Estrategias de afrontamiento ante el estrés y fuentes de recompensa profesional en médicos especialistas de la Comunidad Valenciana. Rev Esp Salud Publica 2002; 76: 595-604.
5. Ríos-Zambudio A, Sánchez-Gascón F, Martínez-Lage JF, Guerrero M. Influence of residency training on personal stress and impairment in family life: analysis of related factors. Med Princ Pract 2006; 15: 276-80.

6. Ríos-Zambudio A, Sánchez-Gascón F, González-Moro L, Guerrero-Fernández M. Formación investigadora durante la residencia MIR. Rev Esp Enferm Dig 2004; 96: 695-704.

7. Zhou GZ, Wong DD, Nguyen LK, Mendelson RM. Student and intern awareness of ionising radiation exposure from common diagnostic imaging procedures. J Med Imaging Radiat Oncol 2010; 54: 17-23.

8. Fernández-Martínez $\mathrm{O}$, Hidalgo-Cabrera C, Martín-Tapia A, Suárez S, García del Río B. Burnout en médicos que realizan guardias en un servicio de urgencias. Emergencias 2007; 19: 116-21.

9. Belloch-García SL, Renovell-Farré V, Calabuig-Alborch JR, Gómez-Salinas L. Síndrome de agotamiento profesional en MIR de especialidades médicas hospitalarias. An Med Interna 2000; 17: 118-22.

10. Rohlfs I, Arrizabalaga P, Artazcoz L, Borrel C, Fuentes M, Valls-Llobet C. Salud, estilos de vida y condiciones de trabajo de los médicos y médicas de Cataluña. Barcelona: Fundación Galatea Centre d'Anàlisis i Programes Sanitaris; 2007.

11. Agencia Europea para la Seguridad y la Salud en el Trabajo. Promoción de la investigación sobre seguridad y salud en el trabajo en la Unión Europea. Bilbao, 2005. URL: http://osha. Europa.eu/es/publications/forum/15. [31.12.2009].

12. Instituto Nacional de Seguridad e Higiene en el Trabajo. VI Encuesta Nacional de Condiciones de Trabajo. Madrid: Ministerio de Trabajo e Inmigración; 2007. URL: http:// www.insht.es/Observatorio/Contenidos/InformesPropios/ 
Desarrollados/Ficheros/Informe_VI_ENCT.pdf. [31.01.2010]. 13. Grupo de trabajo Fundación Galatea. La salud del MIR: guía para tutores y profesionales de los centros sanitarios docentes. Barcelona: Fundación Galatea; 2008.
14. Grupo de trabajo EPINETAC. Estudio y seguimiento del riesgo biológico en el personal sanitario 1996-2002. Madrid: Sociedad Española de Medicina Preventiva, Salud Pública e Higiene; 2005.

Anexo. Gestión de la prevención de riesgos laborales en el colectivo de médicos internos residentes de un hospital secundario (encuesta).

Datos sociodemográficos

1. Fecha de nacimiento

2. Sexo

3. Lugar de procedencia

Datos laborales

4. Año de residencia: R1 R2 R3 R4

5. Especialidad elegida:

1. Atención primaria 2. Especializada 3. Médica 4. Médico-quirúrgica 5. Otras

6. Especialidad elegida: 1. Era una de tus opciones previa al examen MIR 2. No lo era

7. Experiencia laboral de tipo médico anterior a la residencia: 1. Sí 2. No

Actividad hospitalaria

8. En el trabajo diario, ¿tienes pacientes a tu cargo? 1. Sí 2. No

9. Opinas que la supervisión que hacen de tu trabajo es: 1. Buena 2. Regular 3. Mala

10. La relación con tu tutor/a principal con respecto a criterios de formación (actividades y formas de enfocar las rotaciones, participación en la toma de decisiones, tareas, apoyo...) es: 1. Buena 2. Regular 3. Mala

11. ¿Realizas guardias? 1. Sí 2. No

12. ¿De qué tipo? (en este momento)

13. Número de horas que trabajas habitualmente a la semana (incluyendo guardias)

14. La duración de la jornada laboral (tiempo de trabajo) me parece adecuada: 1. Sí 2. No

15. ¿Cómo calificarías el tiempo que llevas de 'residencia' con respecto a... ?

16. ¿Crees que tu programa formativo se está cumpliendo adecuadamente? 1. Sí 2. No

Formación en prevención de riesgos laborales previa a la residencia

17. ¿Tuviste formación de pregrado (durante la carrera) en riesgos laborales? 1. Sí 2. No

18. En caso afirmativo, ¿dónde te la enseñaron?

19. Valora la cantidad de la formación

20. Valora la calidad de la formación

21. En cualquier caso, considero que los conocimientos en prevención de riesgos laborales en el pregrado son útiles o aplicables: 1. Sí 2. No

Información-formación en prevención de riesgos laborales a su llegada al hospital

22. ¿Conoces cuáles son las funciones del Servicio de Prevención del hospital? 1. Sí 2. No
23. En el curso de 'Bienvenida de Residentes' recibiste alguna formación sobre prevención de riesgos laborales: 1 . Sí 2 . No

24. ¿Has acudido al reconocimiento médico de inicio? 1. Sí 2. No

Si has acudido, sigue contestando a las siguientes preguntas:

25. En tu opinión, debería ser: Voluntario Obligatorio

26. ¿Consideras que fue útil? 1. Sí 2 . No

27. ¿Recuerdas si recibiste información-formación sobre prevención de riesgos laborales en el reconocimiento de inicio? 1. Sí 2. No

28. En caso afirmativo, ¿de qué tipo de riesgos te informaron?

29. Dentro del Servicio de Prevención, ¿quién te informó? 1. Enfermera 2. Médico 3. Enfermera y médico

30. ¿Consideras que la cantidad de información recibida fue...? 1. Escasa 2. Suficiente 3. Excesiva

31. ¿Consideras que era el momento adecuado para dártela? 1. Sí 2. No

32. ¿Percibes como útil la información recibida? 1. Sí 2. No

Recuerdo de la información recibida

33. ¿Recuerdas la información que te dieron en el Servicio de Prevención? 1. Sí 2. No

34. Durante el tiempo que has pasado en el hospital, ¿te ha informado alguien más de este tema?

35. ¿Hubieras deseado recibir más información (p. ej., utilización de dosímetros, vacunación...)? 1. Sí 2. No

36. ¿Has tenido que aplicar la información-formación recibida?

37. ¿Has necesitado comunicarte desde el reconocimiento de inicio con el Servicio de Prevención? 1. Sí 2. No

38. En caso afirmativo, ¿cuántas veces?

39. Especifica la causa:

40. ¿Has podido acceder al Servicio de Prevención? 1. Sí 2. No

41. ¿Has sufrido algún accidente laboral en este tiempo? 1. Sí 2. No

42. En caso afirmativo, señala si ha sido por alguna de estas causas

43. ¿Conocías el procedimiento a seguir? 1. Sí 2. No

44. ¿Realizaste parte de accidente? 1. Sí 2. No

45. ¿Quién te informó de cómo actuar?

Percepción del riesgo

46. Por último, nos gustaría completar esta encuesta preguntándote cuál es tu percepción del riesgo, cómo lo vives tú (se incluyen aquí los ítems de la tabla II):

- Percepción del riesgo, consideras que está: Presente (sí) / Ausente (no)

- Condiciones de trabajo (medidas de protección adecuadas, formación e información sobre riesgos, disposición de equipos de protección): Adecuada / Deficiente 